\title{
Artificial signal transduction therapy: a futuristic approach to disease treatment
}

\begin{abstract}
6 ...we highlight the possibility of developing an alternative approach to signal transduction therapy, in which the drugs would be used to generate unnatural cell signaling pathways rather than (or in addition to) affecting the existing ones."
\end{abstract}

\begin{abstract}
Keywords: artificial cell signaling pathways $\bullet$ drug development $\bullet$ protein-protein interactions $\bullet$ signal transduction therapy
\end{abstract}

The most basic functions of the cell such as proliferation, differentiation, metabolism and apoptosis are dependent on signal transduction pathways that determine the response of the cell to various environmental signals [1]. These pathways generally begin by the binding of a ligand to an extracellular receptor, which triggers an intracellular signal cascade. The propagation of the signal within the cell is mediated by various protein-protein interactions, in which enzymes activate or deactivate one another with high specificity. Because numerous diseases such as cancer are associated with dysregulation of these networks, much effort has been devoted to developing drugs that disrupt protein-protein interactions or inhibit enzymes that are involved in abnormal cell signaling pathways $[2,3]$. Herceptin and gleevec [4], for example, are well-known anticancer drugs that inhibit the signaling pathways of receptor tyrosine kinases (RTKs) in different ways. The first is an antibody-based drug that binds the extracellular domain of HER-2, a member of the EGFRs family. It disrupts dimerization of HER-2 with neighboring HER receptors and consequently prevents the activation of intracellular kinases. The second drug is a cell-permeable molecule that directly inhibits the intracellular kinase of the PDGF receptor as well as the activity of ABL or c-kit kinases. Despite their different structures and mechanisms of action, treatment with hereceptin or gleevec eventually results in kinase inhibition, which shuts down an intracellular communication channel that is critical to the survival of the cancer cell. These drugs therefore demonstrate well the effectiveness of signal transduction therapy of cancer [3] that exploits the dependence of cancer cells on fewer, upregulated signaling pathways. In contrast to healthy cells that can minimize the effect of these drugs by activating alternative signaling networks, cancer cells cannot evade this acute interference in their molecular circuitry, which leads to selective cell death [3].

In this editorial paper we highlight the possibility of developing an alternative approach to signal transduction therapy, in which the drugs would be used to generate unnatural cell signaling pathways rather than (or in addition to) affecting the existing ones. These new networks could directly induce, for example, cell death or 'repair' or, alternatively, they could be used to activate various different proteins that naturally exist in the cell, but they are not directly involved in the disease. On a theoretical level, we show that the foundations for realizing such systems have already been laid with the development of synthetic molecules that can connect proteins [5-11] and induce new functions that result from these artificial interactions. The idea of applying such molecules in therapy is further supported by our recent study [12] in which a 'chemical transducer' enabled a growth factor to activate an unrelated enzyme, which resulted in the selective activation of an anticancer prodrug.

Proteins that are involved in signal transduction pathways generally possess binding sites for several proteins. In this way, the binding to one protein can promote the rec-

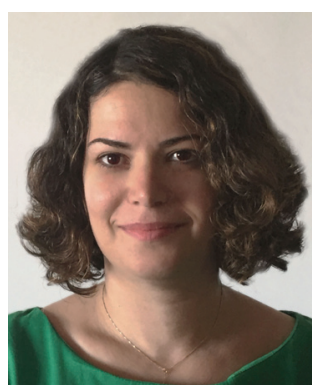

Ronny Peri-Naor

The Weizmann Institute of Science, Herzl St 234, Rehovot 7610001, Israel

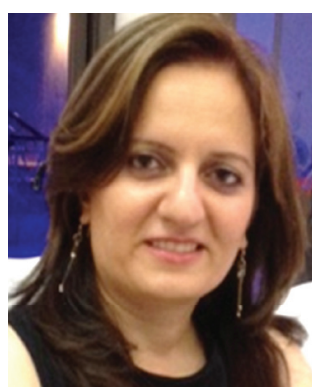

Leila Motiei

The Weizmann Institute of Science, Herzl St 234, Rehovot 7610001, Israel

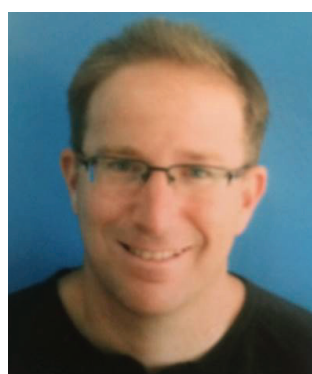

David Margulies

Author for correspondence: The Weizmann Institute of Science, Herzl St 234, Rehovot 7610001, Israel david.margulies@weizmann.ac.il

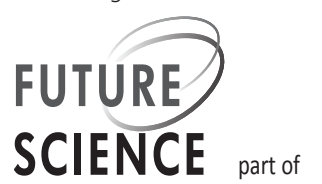


ognition and activation of another, which enables the signal to propagate. In RTKs, for example, the different binding sites are the extracellular receptor and the intracellular kinase [13]. Scaffolding and adaptor proteins [14] lack a catalytic site but can propagate the signal by bringing signaling enzymes in the vicinity of their protein substrates, which results in an effective modification of these proteins. Similarly, various growth factors are homodimers and therefore, they can promote interactions between RTKs, which, in turn, activate intracellular signal transduction processes. According to these principles, different classes of synthetic agents that can bind to several proteins have been developed and used to induce unnatural recognition and signaling events. Chemical inducers of dimerization (CIDs) [5-10], for example, are bivalent compounds that, similar to growth factors, can induce dimerization of cell surface receptors and the consequent downstream signal cascades $[15,16]$. With such systems, the proteins of interest (POIs) are normally fused to an additional binding domain to which a single CID can bind and bring two POIs in proximity. The generality of this approach was demonstrated by the ability of such compounds to induce various protein-protein interactions, control cellular processes as well as by their ability to bind different recognition motifs, [5-10] including short peptide tags [17].

\section{"Whereas in nature growth factors naturally activate their receptor tyrosine kinases binding partners, we have shown how a 'chemical transducer' can make a growth factor trigger the activity of an unrelated enzyme (GST), which corresponds to an unnatural signal transduction step."}

Our expectation that future medicinal chemistry would involve drugs that can rewire natural signaling networks does not rely solely on the properties of CIDs. Whereas CIDs can promote protein-protein interactions and affect cell signaling pathways, this approach generally requires the POIs to be genetically modified, which complicates their use in medicinally relevant applications. One way to circumvent this limitation has been demonstrated with the development of a synthetic agent that, similar to natural adaptor proteins [14], can bind the surfaces of two unmodified POIs [18]. By linking a peptide that binds the Hsc kinase enzyme to another peptide that binds the hDM2 protein, a 'synthetic adaptor protein' [18] was created and used to promote the phosphorylation of hDM2, which is not the physiological substrate of Hsc kinase. Although the function of the chemical adap- tor has so far been demonstrated outside the cell, its ability to induce an unnatural phosphorylation step indicates that artificial cell signaling pathways could be created without changing the natural machinery of the cell [11].

A common feature of the systems above is their ability to bring the desired interacting partners in proximity, which increases their effective molarity [19]. Whereas this principle is frequently applied in natural signal transduction pathways, this is not the only mechanism used to transduce the signal. In the cell, binding interactions between signaling proteins frequently result in allosteric effects that turn the activity of enzymes on and off. Hence, to obtain artificial signaling pathways, the function of signaling enzymes should not be dependent solely on their proximity to substrates - it should also be controlled by the presence of other proteins that activate or inactivate them. In this context, we recently reported a method that enables an enzyme to be activated by another protein that is not its natural binding partner [12]. Unlike CIDs or synthetic adaptors that simultaneously bind two proteins [5-11], our approach relies on a synthetic 'chemical transducer' that can either bind one protein or the other. This transducer consists of a DNA aptamer that binds PDGF and small molecules that inhibit the GST enzyme. In the absence of PDGF, the transducer binds to GST and inhibits its activity. The binding to PDGF, however, makes the two inhibitors less available for binding, which results in the immediate activation of GST. Whereas in nature growth factors naturally activate their RTK binding partners, we have shown how a 'chemical transducer' can make a growth factor (PDGF) trigger the activity of an unrelated enzyme (GST), which corresponds to an unnatural signal transduction step. To demonstrate the relevance of 'chemical transducers' to therapeutic applications, we have also shown that this artificial PDGF-GST communication can change the response of breast cancer cells to treatment with an anticancer prodrug that is activated by GST. Because PDGF is overexpressed in various cancers, this proof-of-principle experiment indicates the potential for obtaining an alternative approach to signal transduction therapy of cancer, in which specific protein biomarkers would be used to activate enzymes that cleave anticancer prodrugs.

We believe that the various systems discussed above demonstrate steps toward achieving a new approach to signal transduction therapy, which would utilize the ability of molecules to generate artificial cell signaling pathways. Note that the proposed approach does not refer to the general concept of controlling biological functions with synthetic molecules that can simultaneously bind different proteins [7]. Instead, such mol- 
ecules must enable one protein to change the activity of another protein, which is not necessarily its natural binding partner, and in a way that would enable the signal to further propagate. Overall, synthetic molecules have already been used to promote signal transduction processes [15], alter the specificity of kinases [18] as well as mediate unnatural enzyme activation and the consequent cleavage of prodrugs [12]. On the other hand, CIDs [8] generally promote interactions between genetically modified proteins, whereas current synthetic 'adaptors' [18] or 'transducers' [12] operate extracellularly. We thus hope that this paper will inspire the development of new synthetic agents that can induce artificial signal transduction steps within natural cells,

\section{References}

1 Hunter T. Signaling - 2000 and beyond. Cell 100(1), 113-127 (2000).

2 Persidis A. Signal transduction as a drug-discovery platform. Nat. Biotech. 16(11), 1082-1083 (1998).

3 Levitzki A, Klein S. Signal transduction therapy of cancer. Mol. Aspects. Med. 31(4), 287-329 (2010).

4 Fischer OM, Streit S, Hart S, Ullrich A. Beyond herceptin and gleevec. Curr. Opin. Chem. Biol. 7(4), 490-495 (2003).

5 Klemm JD, Schreiber SL, Crabtree GR. Dimerization as a regulatory mechanism in signal transduction. Ann. Rev. Immunol. 16(1), 569-592 (1998).

6 Gestwicki JE, Marinec PS. Chemical control over proteinprotein interactions: beyond inhibitors. Comb. Chem. High Throughput. Screen. 10(8), 667-675 (2007).

7 Corson TW, Aberle N, Crews CM. Design and applications of bifunctional small molecules: why two heads are better than one. ACS Chem. Biol. 3(11), 677-692 (2008).

8 Rutkowska A, Schultz C. Protein tango: the toolbox to capture interacting partners. Angew. Chem. Int. Ed. 51(33), 8166-8176 (2012).

9 Fegan A, White B, Carlson JCT, Wagner CR. Chemically controlled protein assembly: techniques and applications. Chem. Rev. 110(6), 3315-3336 (2010).

10 Derose R, Miyamoto T, Inoue T. Manipulating signaling at will: chemically-inducible dimerization (CID) techniques resolve problems in cell biology. Pflugers Arch. Eur. J. Physiol. 465(3), 409-417 (2013). which would bring us closer to achieving artificial signal transduction therapy.

\section{Financial \& competing interests disclosure}

The authors would like to thank the Minerva Foundation, the International Human Frontier Science Project Organization and the European Research Council (Starting Grant 338265) for financial support. The authors have no other relevant affiliations or financial involvement with any organization or entity with a financial interest in or financial conflict with the subject matter or materials discussed in the manuscript apart from those disclosed.

No writing assistance was utilized in the production of this manuscript.

11 Meyer C, Köhn M. A molecular tête-à-tête arranged by a designed adaptor protein. Angew. Chem. Int. Ed. 51(33), 8160-8162 (2012).

12 Peri-Naor R, Ilani T, Motiei L, Margulies D. Protein-protein communication and enzyme activation mediated by a synthetic chemical transducer. J. Am. Chem. Soc. 137(30), 9507-9510 (2015).

13 Lemmon MA, Schlessinger J. Cell signaling by receptor tyrosine kinases. Cell 141(7), 1117-1134 (2010).

14 Bhattacharyya RP, Reményi A, Yeh BJ, Lim WA. Domains, motifs, and scaffolds: the role of modular interactions in the evolution and wiring of cell signaling circuits. Annu. Rev. Biochem. 75(1), 655-680 (2006).

15 Spencer DM, Wandless TJ, Schreiber L, Crabtree GR. Controlling signal transduction with synthetic ligands. Science 262, 1019-1024 (1993).

16 Belshaw PJ, Ho SN, Crabtree GR, Schreiber SL. Controlling protein association and subcellular localization with a synthetic ligand that induces heterodimerization of proteins. Proc. Natl Acad. Sci. USA 93(10), 4604-4607 (1996).

17 Rutkowska A, Haering CH, Schultz C. A FlAsH-based crosslinker to study protein interactions in living cells. Angew. Chem. Int. Ed. 50(52), 12655-12658 (2011).

18 Hobert EM, Schepartz A. Rewiring kinase specificity with a synthetic adaptor protein. J. Am. Chem. Soc. 134(9), 3976-3978 (2012).

19 Hobert EM, Doerner AE, Walker AS, Schepartz A. Effective molarity redux: proximity as a guiding force in chemistry and biology. Isr. J. Chem. 53(8), 567-576 (2013). 